\title{
Natural Deep Eutectics as a "Green" Cellulose Cosolvent
}

\author{
Huy Vu Duc Nguyen, Renko De Vries, and Simeon D. Stoyanov* \\ Cite This: ACS Sustainable Chem. Eng. 2020, 8, 14166-14178 \\ Read Online
}

ABSTRACT: In this study, we report a novel, green chemistry approach for creating new cellulose solvents based on a mixture of a natural deep eutectic solvent (NADES) and a primary cellulose solvent. Because of the strong hydrogen-bond donor and acceptor ability of both the NADES and the primary cellulose solvent, the new mixed system is a cellulose solvent, with an improved cellulose dissolution capacity. We believe that this is a generic approach to prepare an entirely new class of green solvent, capable of dissolving cellulose under mild conditions. This in turn will facilitate the creation of a large amount of new cellulose-based (soft) materials. To illustrate our approach, we show that a NADES based on choline chloride + malic acid can be used as cosolvent for the industrial cellulose solvent $\mathrm{N}$-methylmorpholine- $\mathrm{N}$-oxide mono-

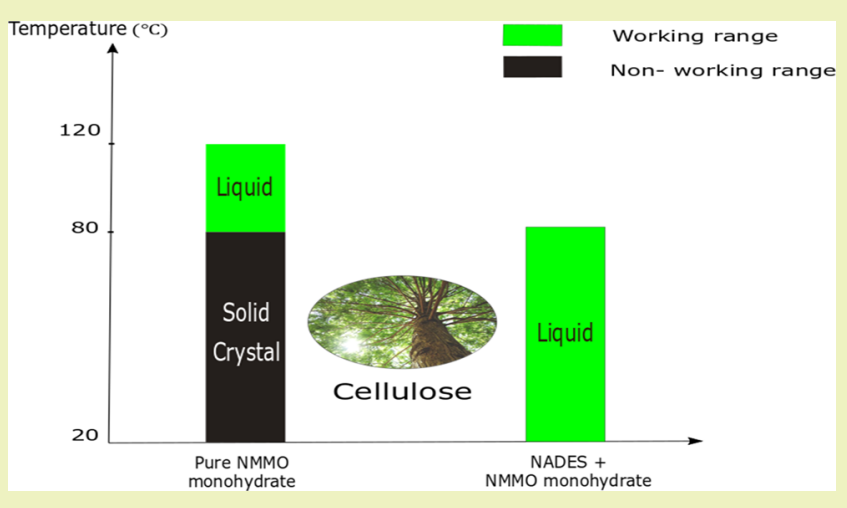
hydrate (NMMO). The new mixed cellulose solvent system has improved cellulose dissolution capacity and has a much broader processing window, which allows working with dissolved cellulose at ambient temperatures, far below $70{ }^{\circ} \mathrm{C}$, where NNMO monohydrate will solidify. This, in turn, can not only help to address the thermal instability issue of pure NMMO when processed at elevated temperatures, but also expands the working conditions of the lyocell process. The recyclability of the solvent system was also conducted with a recovery yield of more than $95 \%$, validating the sustainability of this solvent system. More importantly, the cellulose dissolution capacity was still maintained after the recycling process. To highlight the potential applications, we have created highly anisotropic reconstituted cellulosic suspensions by the solvent exchange process in different antisolvents.

KEYWORDS: cellulose dissolution, natural deep eutectic solvents (NADESs), NMMO monohydrate

\section{INTRODUCTION}

Green material engineering relies on the utilization of biorenewable and biodegradable resources in a sustainable manner with reduced environmental impact. Cellulose being one of the most abundant, biodegradable, environmentalfriendly material on Earth stands out to be an excellent starting material for its applications. ${ }^{1}$ However, many of the important industrial applications require to (selectively) dissolve cellulose. Unfortunately, most of common cellulose solvents are based on harsh chemicals or require significant energy to process, and/or produce byproducts that are not fully environmentally benign.

$\mathrm{N}$-Methylmorpholine- $\mathrm{N}$-oxide (NMMO) has been used successfully to regenerate cellulose into high value-added pure cellulosic materials, by using so called lyocell process. ${ }^{2}$ It is a notable fact that NMMO is significantly less harmful than other cellulose solvents such as concentrated sodium thiocyanate, lithium chloride/dimethylacetamide ( $\mathrm{LiCl} /$ DMAc). ${ }^{3,4}$ As a result, NMMO is quite commonly used when fabricating cellulose-based fibers, films, aerogels, food casings, membranes, beads and sponges. ${ }^{5,6}$

Despite having greater advantages over other classical cellulose solvents, NMMO has also a drawback, regarding its relatively narrow temperature processing window. This is because the NMMO monohydrate melts at $78{ }^{\circ} \mathrm{C}$, while at temperatures higher than $120^{\circ} \mathrm{C}$, cellulose solution in NMMO can undergo a range of strongly exothermic reactions that can cause thermal runaway and extensive degradation. ${ }^{7}$ As a result, number of attempts have been made to find cosolvents that could broaden the temperature window and improve the processability of cellulose dissolution in NMMO. ${ }^{8-10}$ Several aprotic liquids such as dimethyl sulfoxide (DMSO), dimethylformamide and DMAc have been used to mix with NMMO monohydrate for dissolving cellulose. Results of these investigations revealed the reduction of cellulose solubilizing ability of the binary mixture in the whole region of compositions, probably due to the strong intermolecular interaction between NMMO and the aprotic solvent. ${ }^{11}$ Moreover, the addition of DMSO to NMMO monohydrate

Received: July 7, 2020

Revised: August 21, 2020

Published: August 26, 2020 
leads to the rise of exothermicity of cellulose solution. Experimental data shown the negative enthalpies of cellulose dissolving in NMMO-DMSO mixtures, which gives rise to degradation of cellulose solution. ${ }^{11}$ For this reason, DMSO is mainly used as diluent in NMMO technology for fabricating of cellulose film. $^{12}$

A class of solvents that have attracted much interest during these recent years is deep eutectic solvents (DESs). DESs are mixtures of two or more components being associated with each other by means of hydrogen bonding networks, having melting temperatures much lower than those of the individual components. ${ }^{13}$ Natural DESs (NADESs) have drawn much attention as potentially powerful and environmentally friendly solvents for a wide range of processes. ${ }^{14,15}$ Regarding the lignocellulosic biomass processing applications, there have been numerous of ongoing researches on this field. ${ }^{16-18}$ The most well-established mechanism of cellulose dissolution is based on the ability of solvents to disrupt the inter- and intramolecular hydrogen bonding networks in cellulose by forming new H-bonds between solvents and cellulose. ${ }^{19}$ On the other hand, another mechanism governed by hydrophobic interactions due to the amphiphilicity of cellulose has also been reported. ${ }^{20}$ The hypothesis to use NADESs for cellulose solubilization is that NADESs are capable of donating and accepting protons and this characteristic enables the formation of hydrogen bonds with cellulose solute which enhances its solvation properties. Concerning the dissolution of cellulose, so far, they have met with limited success. ${ }^{16,21-23}$ Malic acid (MA)-based NADESs appear to be the most promising group of solvents, which reported a decrease of cellulose crystallinity of up to $20 \%$. $^{24}$

Here, we set out to demonstrate that certain NADES can also act as an alternative cosolvent for NMMO (and possibly other cellulose solvents) and thus forming a new mixed cellulose solvent, which could be better solvent than each of the individual constituents. The logic of using NADES as a cosolvent for NMMO is that they are environmentally benign, extensively hydrogen-bonded, yet have very low crystallization/glass transition temperatures. Therefore, we have hypothesized that they may be able to prevent NMMO from crystallizing while having similar or even better cellulose solubility. The specific NADES that we have used here is based on the choline chloride (CC) + MA (CM) mixtures. We choose $\mathrm{CC}$ as a hydrogen bond acceptor because it is a cheap, biodegradable, and nontoxic quaternary ammonium salt which can be either extracted readily from biomass or massively produced from fossil reserves through a very high atom economy process. ${ }^{25} \mathrm{MA}$, a naturally occurring substance found in apple and pear, is used as a hydrogen bond donor. This acid is widely used as food additives and pharmaceutical ingredients. $^{26}$

The paper is organized as follows: first, we characterize the thermo-physical properties of the NADES CM eutectic mixtures. Next, we show that our investigated NADES CM can incorporate into NMMO monohydrate to some extent to form the new cellulose solvent system, which is a stable supercooled liquid over a wide range of temperatures and maintains its capacity to dissolve cellulose. Finally, we have used our new cellulose solvent to demonstrate that we can prepare reconstituted cellulose particles with various anisotropic shape, by antisolvent precipitation in water and glycerol/water mixture.

\section{MATERIALS AND METHODS}

Materials. Microcrystalline cellulose (MCC) was purchased from Sigma-Aldrich. CC high purity grade, D-L-malic acid high purity grade were both purchased from VWR Life Science. N-Methylmorpholine $\mathrm{N}$-oxide monohydrate (NMMO monohydrate) (98\% purity) was obtained from Alfa Aesar. Mili-Q water was used for all of the experiments.

Preparation of NADESs. NADESs composed of CC as hydrogen bond acceptor and MA as hydrogen bond donor were obtained by vortex mixing two solid components at different molar ratios of 1:1, 1.5:1, 2:1, 1:1.5, 1:2. The components mixture was preheated at 70 ${ }^{\circ} \mathrm{C}$ in an ultrasonic bath (Elma S30H, $37 \mathrm{kHz}, 80 \mathrm{~W}$ ) for $2 \mathrm{~h}$ and then continued mixing under stirring in an oil bath at same temperature to obtain a clear liquid. Next, the prepared NADESs were cooled down to room temperature. We found that the combination of preheating in ultrasonic bath and mixing was much more efficient in obtaining homogenous NADESs than just heating (and stirring) alone. It is such an important point that should be taken under consideration when preparing NADES which has not yet been addressed in the literature.

Characterization of Solvents and Cellulose Solutions. ${ }^{1} \mathrm{H}$ NMR and ${ }^{13} \mathrm{C}$ NMR measurements were performed on a Bruker AVANCE 400 spectrometer operated at room temperature. Spectra were acquired for chemical shifts of CC, MA and resulting NADESs CM. Each ${ }^{1} \mathrm{H}$ NMR spectrum is the average of 32 scans. Samples of approximately $10 \mathrm{mg}$ of each component and eutectic mixture in 1 $\mathrm{mL}$ of deuterium oxide $\left(\mathrm{D}_{2} \mathrm{O}\right)$ were prepared in a thick-walled tube under vortex mixing until they were homogeneously mixed. These solutions were then transferred into the $5 \mathrm{~mm}$ NMR tubes.

Fourier transform Infrared (FT-IR) measurements were carried out using a Bruker FT-IR equipped with OPUS software and an ATR sampling accessory. Spectra were acquired for wavenumber from 400 to $4000 \mathrm{~cm}^{-1}$ with a resolution of $4 \mathrm{~cm}^{-1}$ in transmittance mode. Each spectrum was the average of 32 scans.

Different weight fractions of NADES CM1.5:1 (0-35 wt \%) were incorporated into NMMO monohydrate to form a new cellulose solvent system. In fact, there are all four different molecules that make up this new cellulose solvent system, including NMMO, water, CC, and MA. However, because in this study we have not independently varied the amount of water, we will call our system a ternary solvent system (NCM), referring to the two components of NADES and NMMO, which we have varied independently. This is similar when people refer to NMMO as a cellulose solvent, while they implicitly meaning NMMO monohydrate, which contains $13.3 \mathrm{wt} \%$ of water (anhydrous NMMO is not a cellulose solvent). In reality, the system we described here is quaternary because the water concentration is comparable to those of MA and CC and in addition, it could be varied independently.

Differential scanning calorimetry (DSC) was carried out on a PerkinElmer model DSC 8500. Samples of $30 \mathrm{mg}$ were put into stainless steel DSC pans. Measurements were carried out from -90 to $50{ }^{\circ} \mathrm{C}$ with a heating and cooling rate of $10{ }^{\circ} \mathrm{C} / \mathrm{min}$ with a $\mathrm{N}_{2}$ purge of $20 \mathrm{~mL} / \mathrm{min}$.

Thermogravimetric analysis (TGA) was done using a PerkinElmer model TGA 4000. Samples of approximately $20 \mathrm{mg}$ were used in ceramic crucible. The samples were heated from $30^{\circ} \mathrm{C}$ up to $600{ }^{\circ} \mathrm{C}$ at a heating rate of $10{ }^{\circ} \mathrm{C} / \mathrm{min}$, with a $\mathrm{N}_{2}$ purge of $20 \mathrm{~mL} / \mathrm{min}$.

The water content of the prepared NADES CM, new mixed cellulose solvent (NCM) were measured by Karl Fischer titration method using a Metrohm $890 \mathrm{KF}$ Titrando.

Solubility of MCC in Ternary NMMO-NADES (NCM) Solvent. Prior to use, MCC was dried overnight under vacuum at $50{ }^{\circ} \mathrm{C}$. The solubility of the MCC in ternary solvent system with different compositions were measured by a cloud point method ${ }^{16,18}$ and further monitored by observing the solution under a phase contrast optical microscope. For each solvent composition, $5 \mathrm{~g}$ of solvent was weighed in a glass vial. The glass vials were first fluxed with $\mathrm{N}_{2}$ and capped during the dissolution process to avoid the moisture uptake. Next, $50 \mathrm{mg}$ MCC was added, the mixture was heated to $80{ }^{\circ} \mathrm{C}$ in the oil bath and stirred vigorously until the 
transparent solutions were obtained. The heating temperature was kept constant for all experiments. Consecutive additions of $50 \mathrm{mg}(=1$ wt $\%$ of MCC) were repeated until the solutions remained cloudy or after the maximum stirring time of $10 \mathrm{~h}$ had been reached (which is supposed to the maximum cellulose concentration).

Rheology of MCC in NMMO-NADES Solvent. The rheology of MCC in the ternary NCM solvent was characterized using a MCR 501 Rheometer (Anton Paar, Austria) equipped with cone-plate geometry $\left(4^{\circ}-25 \mathrm{~mm}\right)$ and a Peltier temperature control system. Solutions of various cellulose concentrations were prepared in selected NCM solvent systems by heating the solvent-cellulose mixtures at $80{ }^{\circ} \mathrm{C}$ and stirring at $500 \mathrm{rpm}$ until transparent solutions were obtained. Before measurement, solutions were kept at room temperature, in the desiccator to prevent the moisture and oxygen uptake. Samples were used for measurements within 3 days after their preparation. Shear rate sweeps were obtained by varying shear rate from 0.01 to $500 \mathrm{~s}^{-1}$ and measuring the viscosity, for a range of temperatures from 20 to $80{ }^{\circ} \mathrm{C}$. Frequency sweeps where performed for oscillation frequencies between 0.01 and $100 \mathrm{rad} / \mathrm{s}$ at a fixed low strain amplitude of $\gamma=1 \%$, within the linear viscoelastic regime. Temperatures were varied between 20 and $80{ }^{\circ} \mathrm{C}$. For both shear rate sweeps and frequency sweeps, to prevent moisture and oxygen uptake, a thin layer of low viscosity tetradecane was applied to the edge of the measuring cell.

Antisolvent Precipitation. Mili- $Q$ water and glycerol/Mili- $Q$ water mixtures $(70 / 30 \% \mathrm{w} / \mathrm{w})$ were used as antisolvents for cellulose regeneration. A syringe with $10 \mathrm{~mL}$ of 2 wt \% MCC dissolved in a ternary solvent, was gradually added into the beaker of antisolvent with injection rate $10 \mathrm{~mL} / \mathrm{min}$ to precipitate the dissolved cellulose.

Recycling of Ternary Cellulose Solvent. The collected mixture after antisolvent precipitation process by water were subjected to distillation for $2 \mathrm{~h}$ at $50{ }^{\circ} \mathrm{C}$ under vacuum using a rotary evaporator. The recovery yield was calculated by measuring the mass differences of recycled ternary solvent compared to the original used ternary solvent. This process was repeated in 5 times.

$$
\text { Recovery yield }(\%)=\frac{m_{\text {recycled solvent }}}{m_{\text {original solvent }}} \times 100
$$

Confocal Scanning Laser Microscopy. Confocal laser scanning microscope (Nikon C2) was used to characterize the microstructures of precipitated cellulose. An aliquot $(40 \mu \mathrm{L})$ of staining solution containing $0.1 \%$ Calcofluor white was added to $1 \mathrm{~g}$ of cellulose sample followed by gently mixing, and the sample was then washed with Mili$\mathrm{Q}$ water to remove the excess stain. Stained samples were applied to single-well slides, and a coverslip was placed carefully on the microscope slide. Samples were excited with laser beam at $405 \mathrm{~nm}$ while the emission was also measured at $405 \mathrm{~nm}$. Samples were observed under the microscope using $4 \times$ and $20 \times$ magnification.

X-ray Diffraction. X-ray diffraction (XRD) spectra for scattering angles of $2 \theta=5-60^{\circ}$ were obtained using a Bruker D8 ADVANCE $\mathrm{XRD}$ diffractometer. A Cu K $\alpha$ radiation source $(\lambda=0.15418 \mathrm{~nm})$ was used at a voltage of $40 \mathrm{kV}$ and a current of $40 \mathrm{~mA}$. The crystallinity index (CrI) for the native MCC and regenerated cellulose was calculated based on the "Segal method". ${ }^{27}$ In this approach, CrI is calculated from the ratio between the intensity of the crystalline peak $\left(I_{\mathrm{t}}-I_{\mathrm{am}}\right)$ to the total intensity $\left(I_{\mathrm{t}}\right)$ by the following equation.

$$
\mathrm{CrI}=\frac{I_{\mathrm{t}}-I_{\mathrm{am}}}{I_{\mathrm{t}}} \times 100(\%)
$$

where $I_{\mathrm{t}}$ is the diffraction intensity of the main crystalline peak (200) of cellulose $\mathrm{I} \beta$ at $2 \theta \approx 22.6^{\circ}$ and the peak (020) for cellulose II at $2 \theta$ $\approx 21.8^{\circ}, I_{\text {am }}$ is the diffraction intensity of the amorphous cellulose at $2 \theta \approx 18^{\circ}$ for cellulose $\mathrm{I} \beta$ and at $2 \theta \approx 16^{\circ}$ for cellulose $\mathrm{II}^{28}$

The lattice spacing ( $d$-spacing) $\left(d_{h, k, l}\right)$ and the crystallite sizes $\left(L_{h, k, l}\right)$ of native cellulose and regenerated cellulose are determined by using Bragg eq 2 and Scherrer's eq 3, respectively. ${ }^{29}$

$$
d_{h, k, l}=\frac{\lambda}{2 \sin \theta}
$$

$$
L_{h, k, l}=\frac{0.9 \lambda}{\beta \cos \theta}
$$

where $d_{h, k, l}$ is the distance between atomic plane, $\lambda$ is the wavelength of $\mathrm{x}$-ray source $(\lambda=0.15418 \mathrm{~nm}), \theta$ is the scattering angle, $L_{h, k, l}$ is the crystallite size, $\beta$ is the angular peak width at half maximum intensity (FWHM).

\section{RESULTS AND DISCUSSION}

NADESs of CC and MA were obtained by mixing the solid (powdered) ingredients at different CC/MA molar ratios. Sample names for the NADESs are CM1:1 for the 1:1 molar ratio, and so forth. Transparent clear liquids were obtained by a combined preheating in ultrasonic bath and mixing. These transparent liquids were kept at room temperature for 60 days after which their visual appearance was recorded. Results are given in Table 1.

\section{Table 1. Visual Characterization of Obtained NADESs}

$\begin{array}{lll}\begin{array}{c}\text { molar } \\ \text { ratio }\end{array} & \text { abbreviation } & \begin{array}{c}\text { visual appearance } \\ \text { (after } 60 \text { days at room temperature) }\end{array} \\ 2: 1 & \text { CM2:1 } & \text { mostly clear liquid, some crystallites } \\ 1.5: 1 & \text { CM1.5:1 } & \text { transparent, clear liquid } \\ 1: 1 & \text { CM1:1 } & \text { transparent, clear liquid } \\ 1: 1.5 & \text { CM1:1.5 } & \text { turbid white liquid } \\ 1: 2 & \text { CM1:2 } & \text { solid }\end{array}$

After having stored at room temperature for 60 days, only the NADESs CM1:1 and CM1.5:1 had remained transparent clear liquids. All other mixtures with molar ratios CC/MA further away from stoichiometry had either crystallized or had become turbid. To demonstrate that during the NADES preparation no chemical reactions had taken place, ${ }^{1} \mathrm{H}$ NMR spectra were acquired for both the individual NADES components and the representative CM1:1 NADES. Results are shown in Figure 1. The NMR spectra of the NADES is an exact superposition of the spectra of the components, indicating the no chemical reactions had taken place during the NADES preparation. According to Rodriguez Rodriguez et al., ${ }^{30}$ the stability of DES for further applications need to be taken into account attributed to the degradation and also the formation of ester during the preparation procedure. From the ${ }^{1} \mathrm{H}$ NMR and ${ }^{13} \mathrm{C}$ spectra (Supporting Information, Figures S1 and S2) no evidence of additional peaks formed by the esterification reactions were found.

Indications for the establishment of a network of hydrogen bonds in the CM1:1 NADES was sought in FT-IR spectra. The FT-IR spectra of CC and MA, and the NADES CM1:1, showing the $\mathrm{OH}$ stretching and carbonyl stretching regions, are compared and shown in Figure 2. Two characteristic changes in the spectrum of the NADES as compared to that of the pure components are typical features for the formation of a network of hydrogen bonds. First, the pure components have sharp -OH stretch bands at $3214 \mathrm{~cm}^{-1}$ (CC) and $3442 \mathrm{~cm}^{-1}$ (MA), but in the CM1:1 NADES, these peaks are replaced by the broad bands below $3300 \mathrm{~cm}^{-1}$. The trait of broad $\mathrm{OH}-$ group in NADES is believed to be the outcome of a more intensive hydrogen bonding network. ${ }^{31}$ In addition, the sharp superimposed bands in the pure components are no longer existent in the eutectic mixture. The complexity of these broad bands makes it difficult to interpret, however, these results are in good agreement with previous studies, suggesting the presence of multiple hydrogen bonding network in the form of 


\section{choline chloride}
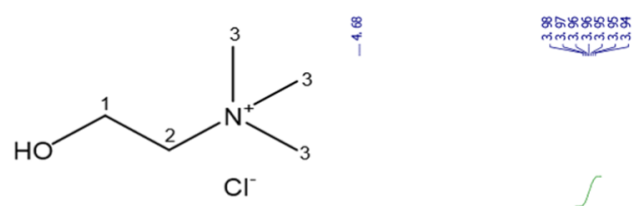

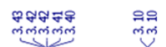

$\mathrm{Cl}$

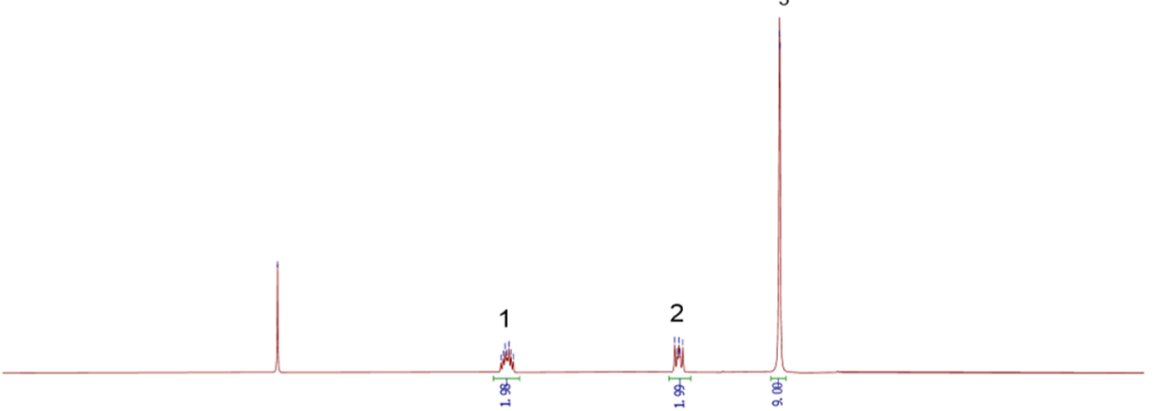

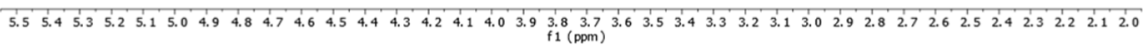

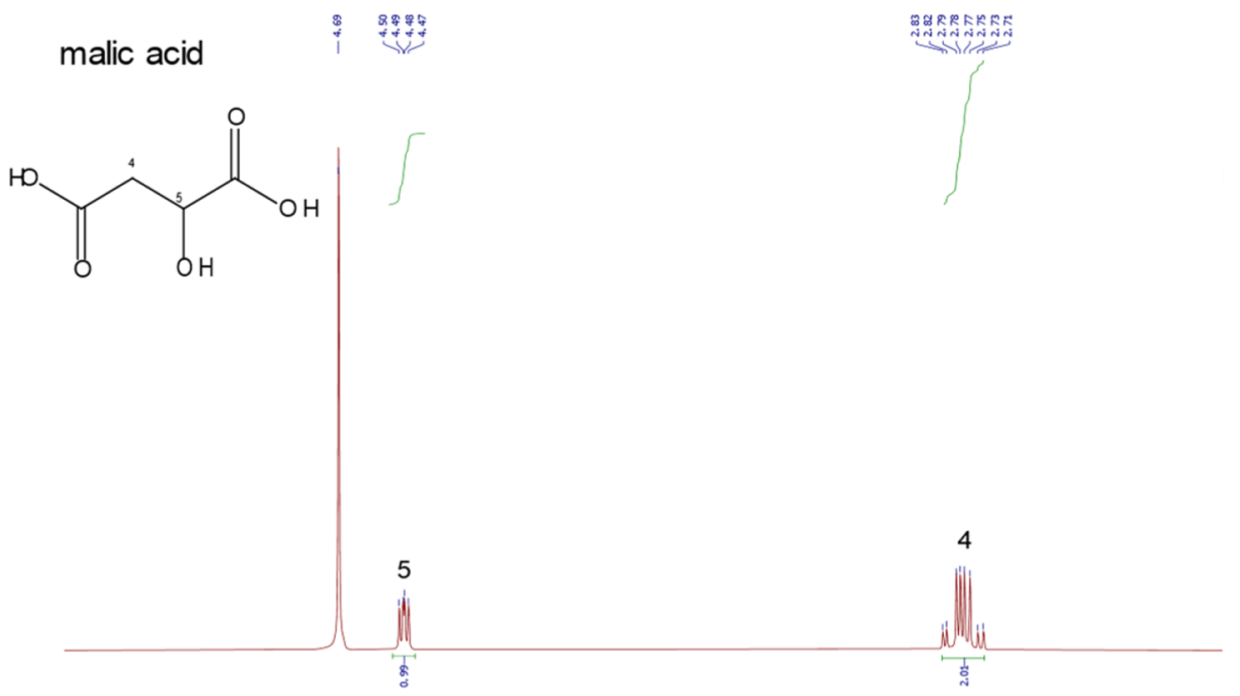

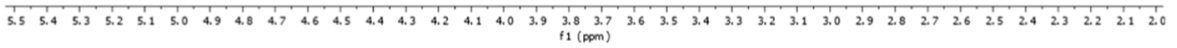

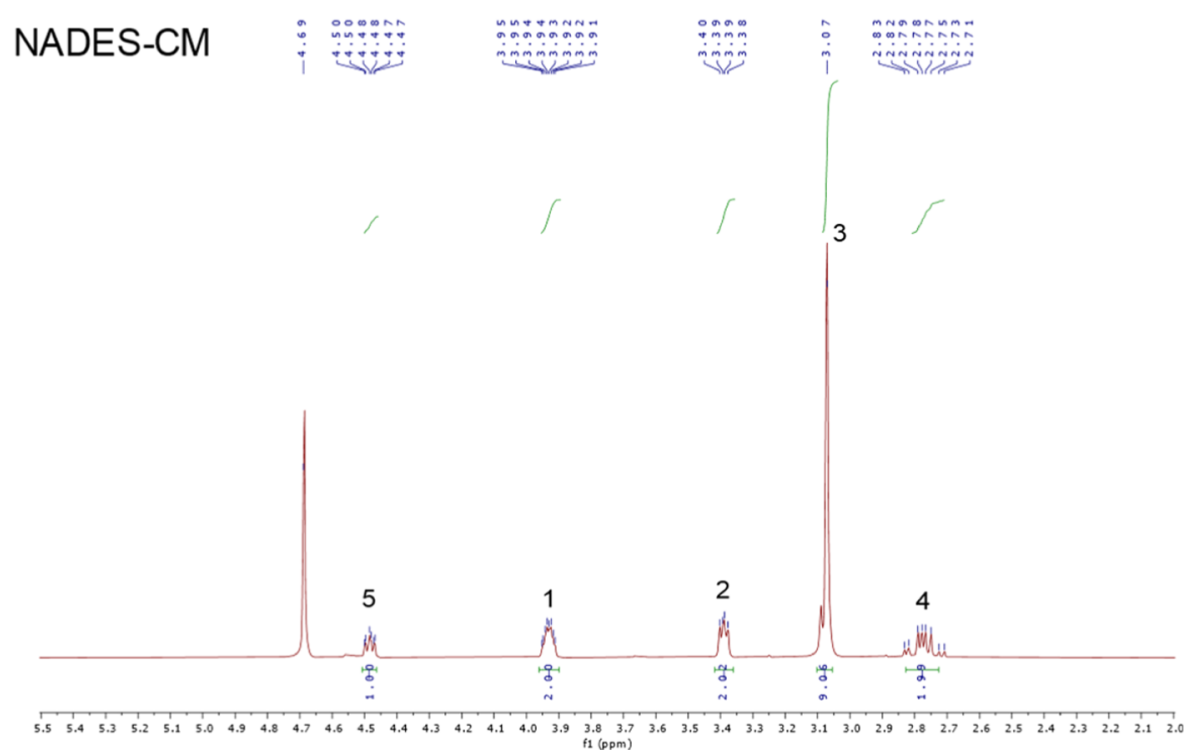

Figure 1. ${ }^{1} \mathrm{H}$ NMR spectra of CC, MA, and the CM1:1 NADES. 

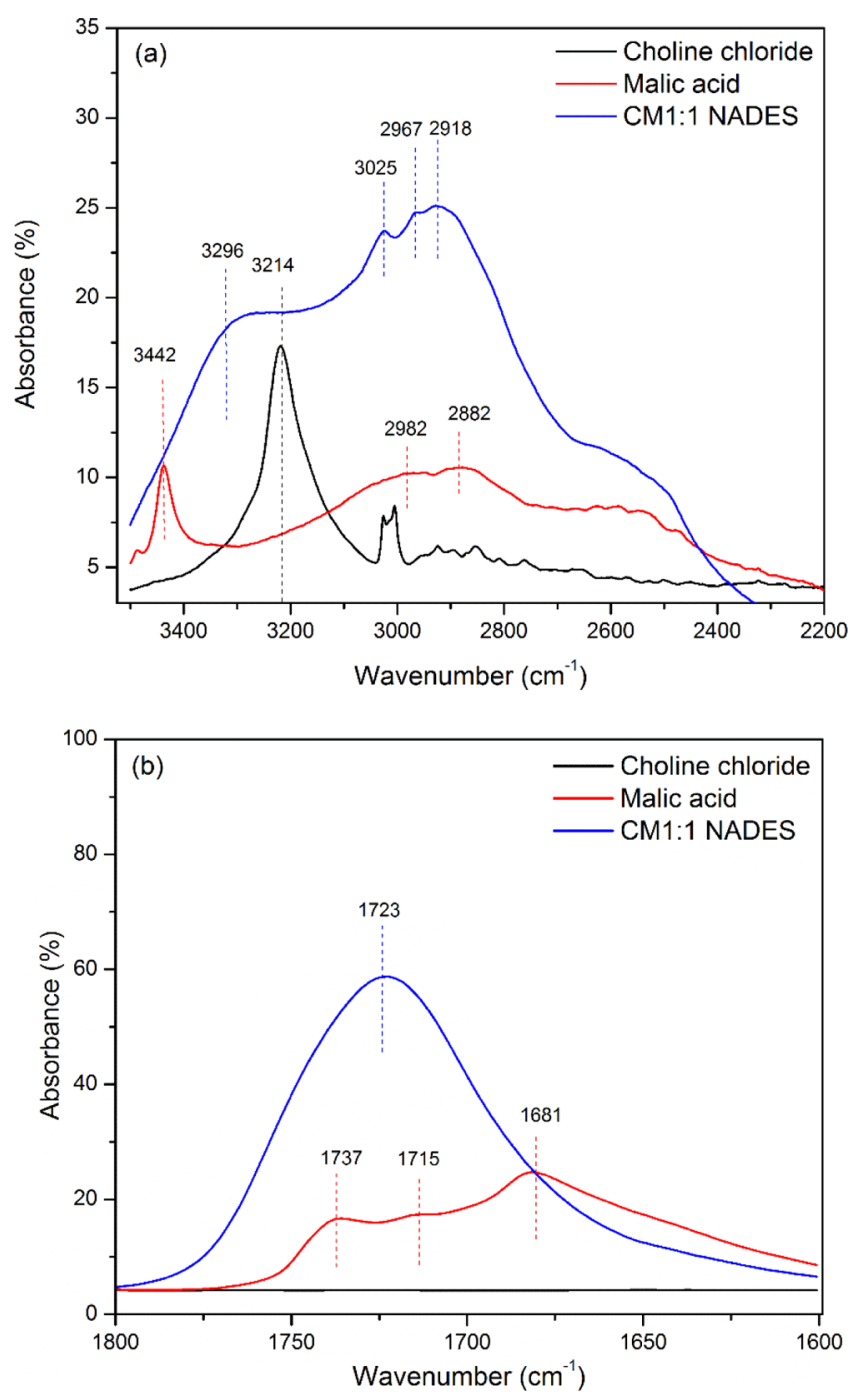

Figure 2. FT-IR spectra of pure CC, MA, and the CM1:1 NADES in the $\mathrm{OH}$ region (a) and carbonyl stretch region (b).

$\mathrm{OH}$ (MA) $\cdots \mathrm{Cl}^{-}, \mathrm{OH}$ (choline) $\cdots \mathrm{Cl}^{-}$, and MA dimers. ${ }^{32}$ Second, the stretch bands for the MA hydrogen donor carbonyl group $\mathrm{C}=\mathrm{O}$ represented by two broad bands, originally located at wavenumbers of 1737 and $1681 \mathrm{~cm}^{-1}$ together with a weaker mode at $1715 \mathrm{~cm}^{-1}$. The two former dominant bands can be assigned to the nonhydrogen bonded carbonyl groups. While in the eutectic mixtures, these bands have transformed to a broad peak at wavenumber of approximately $1723 \mathrm{~cm}^{-1}$. This band can be assigned to carbonyl groups hydrogen bonded to $\mathrm{OH}$ groups. This result revealed the formation of deep eutectic mixtures via hydrogen bonding network, which have been confirmed in previous experimental and computational studies. ${ }^{32,33}$

Next, the thermal properties of the NADESs at different CC/MA ratios were investigated using TGA and DSC. Representative DSC curves and TGA thermograms (for the CM1:1 NADES) are shown in Figure 3. The DSC curves were analyzed to give the glass transition temperatures $T_{\mathrm{g}}$ of systems at different CC/MA ratio's (see example for CM1:1 in Figure $3 \mathrm{a})$. The TGA thermograms were analyzed to give the onset of decomposition temperatures $T_{\mathrm{dec}}$ of systems at different CC/ MA ratio's (see example for CM1:1 in Figure $3 \mathrm{~b}$ ). The
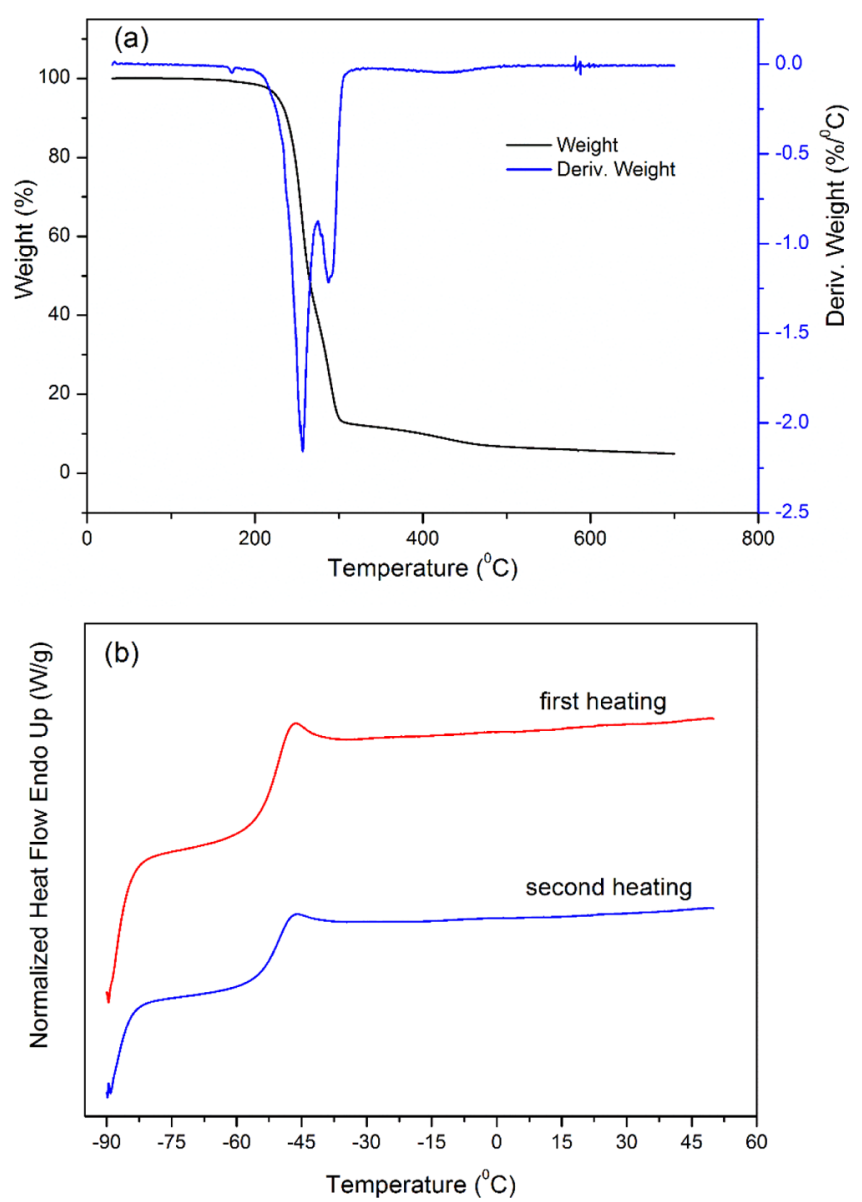

Figure 3. TGA (a) and DSC (b) analysis of the resulting CM1:1 NADES.

resulting glass transition and onset of decomposition temperatures are given in Table 2.

Table 2. Thermal Properties of NADESs Deduced from DSC and TGA: Glass Transition Temperature $\left(T_{\mathrm{g}}\right)$ and the Onset of Decomposition Temperature $\left(T_{\mathrm{dec}}\right)$

\begin{tabular}{lcc} 
NADES & $T_{\mathrm{g}}\left({ }^{\circ} \mathrm{C}\right)$ & $T_{\text {dec }}\left({ }^{\circ} \mathrm{C}\right)$ \\
CM2:1 & -60.79 & 248.71 \\
CM1.5:1 & -57.19 & 220.33 \\
CM1:1 & -55.26 & 211.73 \\
CM1:1.5 & -45.74 & 207.45 \\
CM1:2 & -40.82 & 206.33 \\
\hline
\end{tabular}

From the DSC measurements, we find no evidence of melting temperature for both first and second heating cycle. Instead, the DSC curves clearly show a glass transition temperature $T_{\mathrm{g}}$ of around $-50{ }^{\circ} \mathrm{C}$. Deep eutectic systems are often introduced as systems for which, because of charge delocalization formed by hydrogen bonding, the melting point of the crystal phase of the mixture is lower than the crystal phases of the components but it is in fact not uncommon for NADES systems to exhibit glass transitions that preclude crystallization. ${ }^{34}$ The onset of decomposition temperatures $T_{\text {dec }}$ that we find for the $\mathrm{CM}$ systems are $200-250{ }^{\circ} \mathrm{C}$ and increase with increasing $\mathrm{CC}$ content. The low glass transition temperatures and the high decomposition temperatures imply that the CM system that we have introduced here is a 
chemically and physically supercooled liquid over a wide range of temperature. A similar characteristic was highlighted in sugar-based low melting mixtures in previous study. ${ }^{35}$

Next, we consider the properties of the CM1.5:1 NADES as a cosolvent for NMMO monohydrate. A ternary solvent system consisting of NMMO. $\mathrm{H}_{2} \mathrm{O}$ with $25 \%$ of CM1.5:1 (w/ w) is abbreviated as NCM $25 \%$. DSC curves and TGA thermograms for NCM solvents with different percentages of CM1.5:1 are shown in Figure 4.
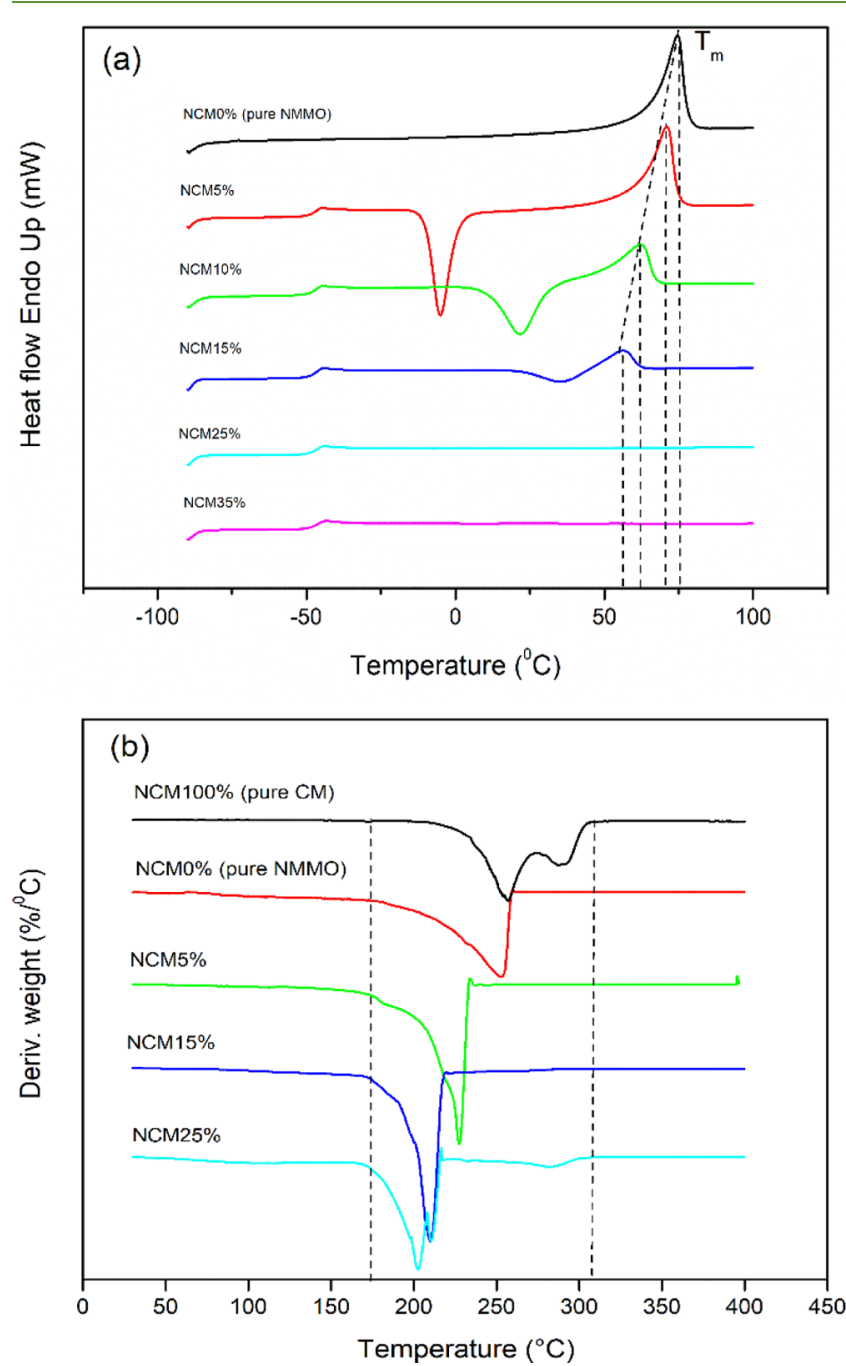

Figure 4. Thermal analysis of ternary NMMO $\cdot \mathrm{H}_{2} \mathrm{O}+\mathrm{CM} 1.5: 1$ solvents of different weight fractions of CM1.5:1 (a) DSC, heat flow $(\mathrm{mW})$ vs temperature $T\left({ }^{\circ} \mathrm{C}\right)(\mathrm{b})$ DTG, temperature derivative $\mathrm{d} / \mathrm{d} T$ $\left(\mathrm{m} / \mathrm{m}_{0}\right)$ of weight $\left(\% /{ }^{\circ} \mathrm{C}\right)$ vs temperature $T$. Weight fraction of CM1.5:1 is indicated above the corresponding curves.

DSC heating curves for NCM solvents with different weight fractions of CM1.5:1 (0-35 wt \%) are shown in Figure 4a. The NMMO monohydrate (NCM0\%) exhibits a single melting peak at around $75{ }^{\circ} \mathrm{C}$. This is the main melting temperature of crystalline NMMO monohydrate. At low weight fractions, the addition of CM1.5:1 lead to a gradual decrease of the melting point of NMMO monohydrate. For weight fractions up to $15 \%$ wt of CM1.5:1 a melting peak can still be observed, but for a weight fraction of $25 \%$ wt or higher, we no longer detected melting peaks (upon heating) or crystallization peaks (upon cooling). At nonzero CM1.5:1 small peak appears for the ternary solvents at around $-50{ }^{\circ} \mathrm{C}$ which attributes to the glass transition of the ternary solvent. The unusual properties of NADES are governed by hydrogen bonding network. In general, compounds capable of donating or accepting electrons or protons to form hydrogen bonds show high solubility. ${ }^{34}$ In this study, we found that the investigated DES CM can dissolve NMMO to form new liquid system. The depression of the melting temperature of NMMO monohydrate upon mixing with NADES may arise from the development of intensive intermolecular interactions between the components, indicating that this is possibly a ternary eutectic system.

TGA analysis of the ternary solvents is shown in Figure $4 \mathrm{~b}$. The degradation peak shift to somewhat lower temperatures at higher weight fractions of CM1.5:1, but even for NCM25\%, there is no degradation for temperatures below $170{ }^{\circ} \mathrm{C}$, which implies that even the NCM $25 \%$ ternary solvent can still be used at reasonably high temperatures, which may be helpful for the more rapid dissolution of solutes.

The solubility assay of MCC in the NCM ternary solvents was carried out by stirring solvent $\mathrm{NCM}$ at $80{ }^{\circ} \mathrm{C}$ to which powdered MCC was added in a stepwise fashion. Results are shown in Figure 5.

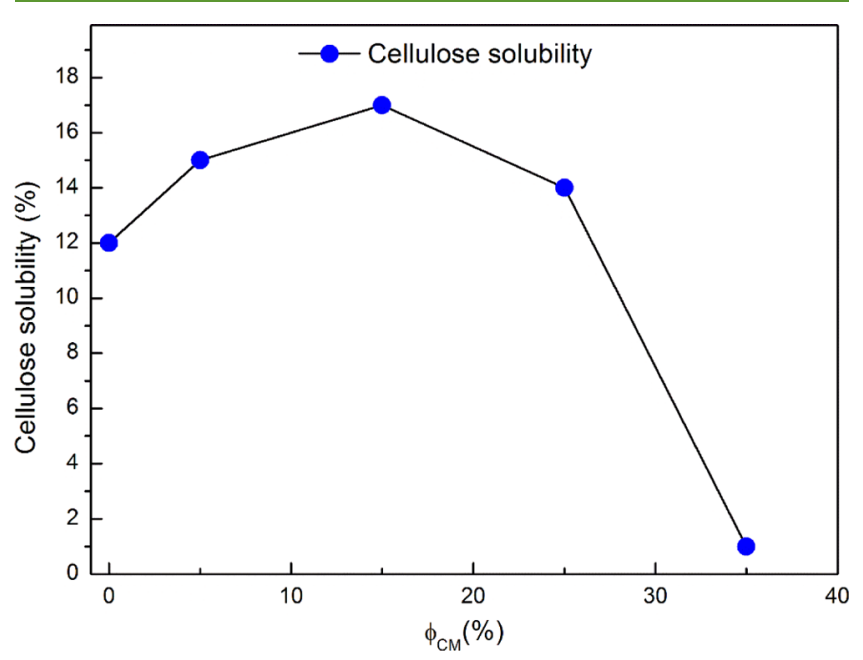

Figure 5. Solubility (\%w/w) of MCC in NCM ternary solvents at 80 ${ }^{\circ} \mathrm{C}$, vs the weight fraction $\phi_{\mathrm{CM}}(\%)$ of the NADES component CM1.5: 1 in the NCM ternary solvent.

We verified experimentally the synergistic effect when incorporating NADES CM with NMMO monohydrate for cellulose dissolution capacity. We find that certain amounts of CM1.5:1, when mixed with NMMO monohydrate (up to 25 wt \%), promote the dissolution of cellulose. Cellulose concentration in ternary solvent NCM increase by $41.6 \%$ than that of in pure NMMO monohydrate when substitute 15 wt \% of NMMO monohydrate by NADES CM. However, with a larger weight fractions of CM1.5:1, the solubility of the MCC in the ternary solvent decreases with increasing CM1.5:1 content. The ternary solvent NCM25\%, with a weight fraction of 25 wt \% of CM1.5:1, appears to be optimal as the cellulose solubility has not decreased significantly while at the same time crystallization of NMMO is completely suppressed. A similar evolution of cellulose dissolution capacity was found in previous effort to utilize various dipolar aprotic solvents to combine with ionic liquid, 1-ethyl-3-methylimidazolium acetate $[\mathrm{EMIm}][\mathrm{OAc}]{ }^{36}$ For the sake of completeness, we 
(a)

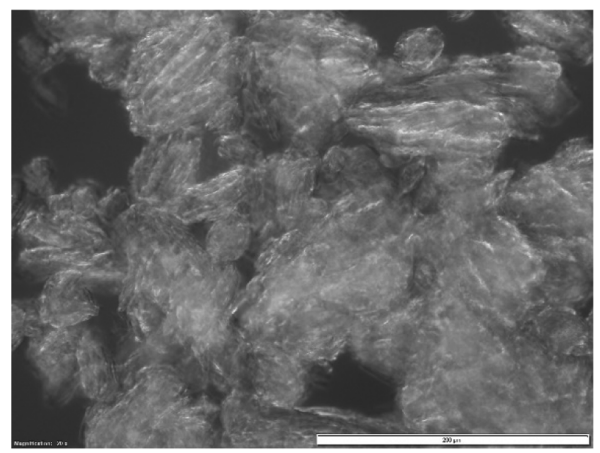

(b)

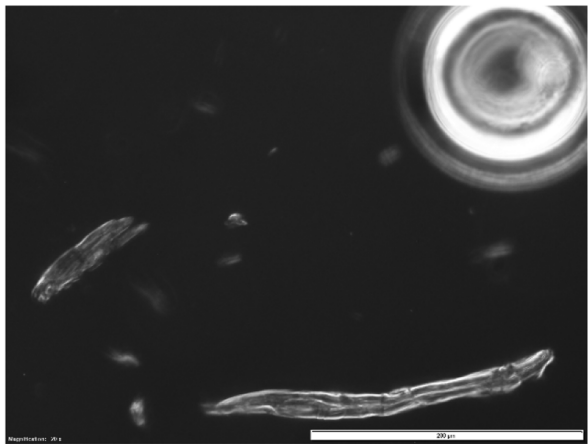

(c)

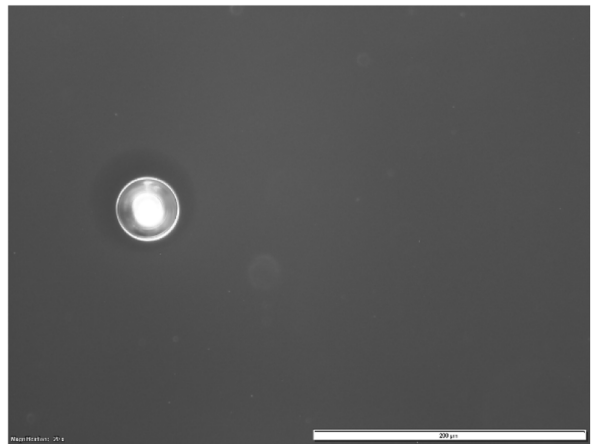

Figure 6. Phase contrast optical microscopy images (20x) of $10 \mathrm{wt} \% \mathrm{MCC}$ in NCM25\% (a) before dissolution when the solvent is introduced, (b) after $30 \mathrm{~min}$, and (c) after dissolution.

have checked explicitly that the addition of cellulose does not induce crystallization either. Figure S3 shows that the DSC thermogram for $2 \%$ of MCC in NCM $25 \%$ is virtually the same as that of the solvent NCM25\% alone. According to these DSC thermograms, no phase transition was observed during cooling even at temperatures below the melting point of NMMO monohydrate. This is, in contrast to previous studies on the thermal behavior of cellulose in NMMO monohydrate which indicated that the recrystallization of NMMO monohydrate itself occurred in dilute cellulose concentrations. ${ }^{37,38}$ More importantly, the new ternary solvent system NCM25\% does not give rise to the exothermic reaction when dissolving cellulose, which resulted in degradation of cellulose solution. This phenomenon was observed when incorporating aprotic solvents with NMMO monohydrate in other aprotic solvents, for instance, DMSO-NMMO monohydrate mixture. ${ }^{11}$ In summary, ternary solvent NCM25\% has not only improved cellulose solubility compared to that of in NMMO monohydrate but also exhibited a much wider operating temperature range. The addition of NADES CM addresses the thermal instability of NMMO monohydrate when operating at elevated temperatures, and prevents the recrystallization of NMMO monohydrate at low temperatures. ${ }^{37}$ Moreover, the use of NADES CM as cosolvent for NMMO monohydrate does not lead to exothermic reactions similar to other investigated aprotic solvents as in previous studies. ${ }^{11}$

The dissolution of cellulose was observed by phase contrast optical microscopy. The samples were taken to observe under a microscope in three different sets: before dissolution, after 30 min of dissolution and after dissolution, respectively. These images are shown in Figure 6. The behavior of cellulose dissolution in ternary solvent NCM is similar to that of
NMMO monohydrate. ${ }^{39}$ Before dissolution, these cellulose particles tend to agglomerate into condensed phase, with many cellulose particle stick together (Figure 6a). After $30 \mathrm{~min}$ of dissolution, these cellulose fibers are disintegrated into small fragments or elongated into rod-like particles (Figure 6b). This behavior confirmed the fast and complete dissolution behavior mode of cellulose into our new ternary solvent NCM. ${ }^{39}$ The dissolution process is then completely accomplished by the disappearance of cellulose particles (Figure 6c).

Solubility behavior of cellulose in NMMO is strongly dependent on the amount of water presence in the NMMO/ water/cellulose system. ${ }^{40}$ Anhydrous NMMO cannot solubilize cellulose, whereas NMMO monohydrate $(\approx 13.3 \%$ water $)$ is considered as the best composition for cellulose dissolving process. For this reason, we have checked the water content in the NADES CM and ternary solvent system NCM25\% by Karl Fischer titration method. The amount of water in NADES CM is relatively low, roughly $0.9 \%$, while the water content in ternary solvent is about $10.3 \%$, which is comparable to what we calculated theoretically. It is also noticeable to mention that we can obviously add more water into ternary solvent system to obtain the right amount of water as in NMMO monohydrate while at the same time retain the dissolution capacity.

Next, we investigate the rheology of MCC dissolved in the NCM25\% solvent. Both the solvent viscosity and the cellulose solution rheology are crucial when using the new solvent for regeneration purposes. The solution rheology also informs about whether dissolution has occurred down to molecular scales, or that dissolution is partial in the sense that cellulose chains still form clusters in the ternary solvent.

The viscosity of NCM ternary solvent systems, as well as the viscosity of the CM1.5:1 NADES as a function of temperature 
are shown in Figure 7. At the same temperature, viscosity of the ternary NCM solvent is much lower than that of the

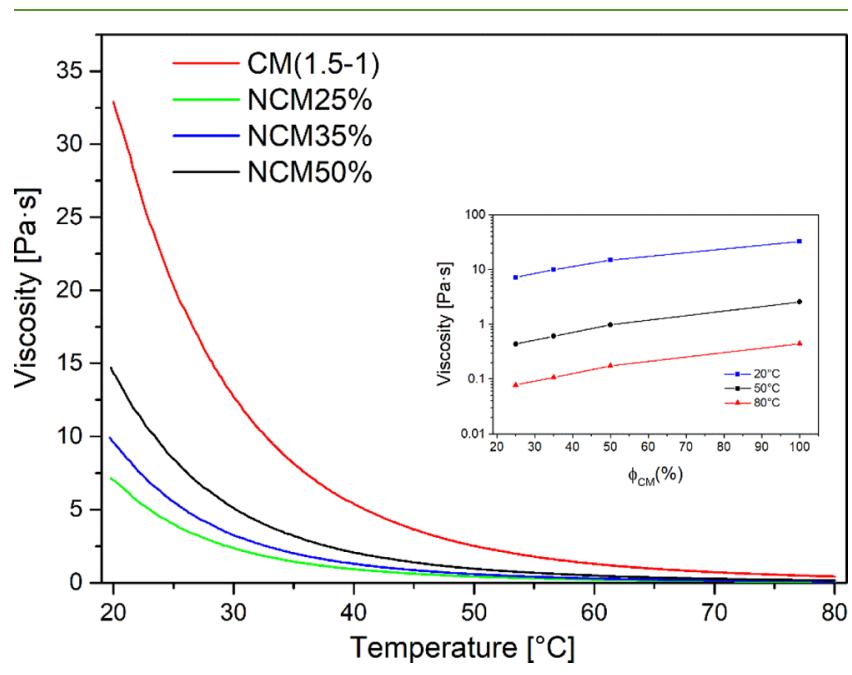

Figure 7. Viscosity $\eta[\mathrm{Pa} \mathrm{s}]$ of the CM1.5:1 NADES and different NCM ternary solvents as a function of temperature $T\left[{ }^{\circ} \mathrm{C}\right]$ at shear rate $10 \mathrm{~s}^{-1}$. The inset figure shows the viscosity vs the weight fraction $\phi_{\mathrm{CM}}(\%)$ of the NADES component CM1.5:1 in the NCM ternary solvent.

representative NADES (CM1.5:1). The high amount contribution of CM in NCM leads to an increase of viscosity in general. The phenomenon of decrease of viscosity can be attributed primarily to the presence of NMMO in NCM solvent system. The intercalation of small NMMO molecule could loosen the bonding strength among complex CM network. Also, the ternary solvent viscosity decreases rapidly with increasing temperature, coming down to around $0.08 \mathrm{~Pa} \mathrm{~s}$ at $80{ }^{\circ} \mathrm{C}$. This is comparable to the viscosity of some chloridebased ionic liquids reported by several studies. ${ }^{41,42}$

Figure 8 shows viscosity as a function of shear rate for NCM25\% ternary solvent for a range of temperatures from 20 to $80^{\circ} \mathrm{C}$. It can be seen clearly that the solvent system behaves similar to a Newtonian liquid at different temperatures. Flow curves for $0.1-2$ wt \% solutions of MCC in NCM25\% are also shown in Figure 8, for temperatures $T=20{ }^{\circ} \mathrm{C}$ (Figure 8a), 40 ${ }^{\circ} \mathrm{C}$ (Figure 8b), $60{ }^{\circ} \mathrm{C}$ (Figure $8 \mathrm{c}$ ), and $80{ }^{\circ} \mathrm{C}$ (Figure $8 \mathrm{~d}$ ). These show the usual behavior for polymer solutions: viscosities decrease with increasing temperature mainly because of the decrease of the solvent viscosity. At higher concentrations, above a critical shear rate, the polymer solutions are shear thinning presumably either because of chain stretching, alignment, or disentanglement. ${ }^{43}$ The critical shear rate above which shear thinning occurs moves to lower shear rates at lower temperatures. This is expected because this critical shear rate is inversely proportional to the segment relaxation time, which in turn is proportional to the solvent viscosity.

From the flow curves we have obtained zero-shear viscosities by extrapolation, and these can be analyzed to obtain the intrinsic viscosity of the cellulose chains in the NCM25\% solvent. The specific viscosities $\eta_{\text {sp }}$ scaled by the cellulose
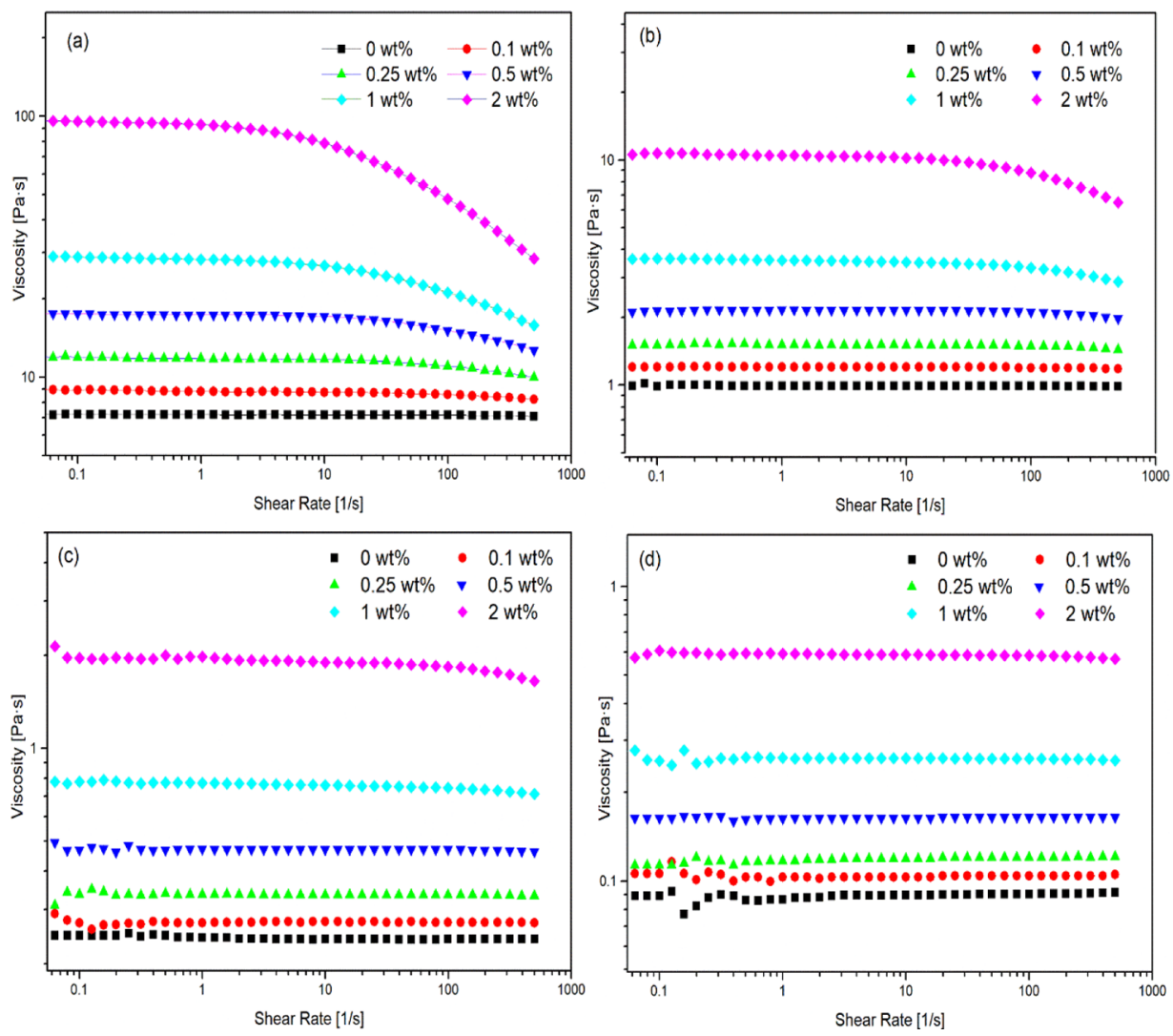

Figure 8. Flow curves for MCC dissolved in the NCM25\% ternary solvent, at a) 20, (b) 40, (c) 60, and (d) $80{ }^{\circ} \mathrm{C}$. Cellulose concentrations are: 0 wt \% (black-filled squares), $0.1 \mathrm{wt} \%$ (red-filled circles), $0.25 \mathrm{wt} \%$ (green-filled triangles), $0.5 \mathrm{wt} \%$ (blue inverted filled triangles), 1 wt \% (turquoise-filled diamond symbols), and 2 wt \% (pink-filled diamond symbols). 
concentration $c$, as a function of $c$, are shown in Figure 9. The intercept of these curves with the $y$-axis is the intrinsic viscosity

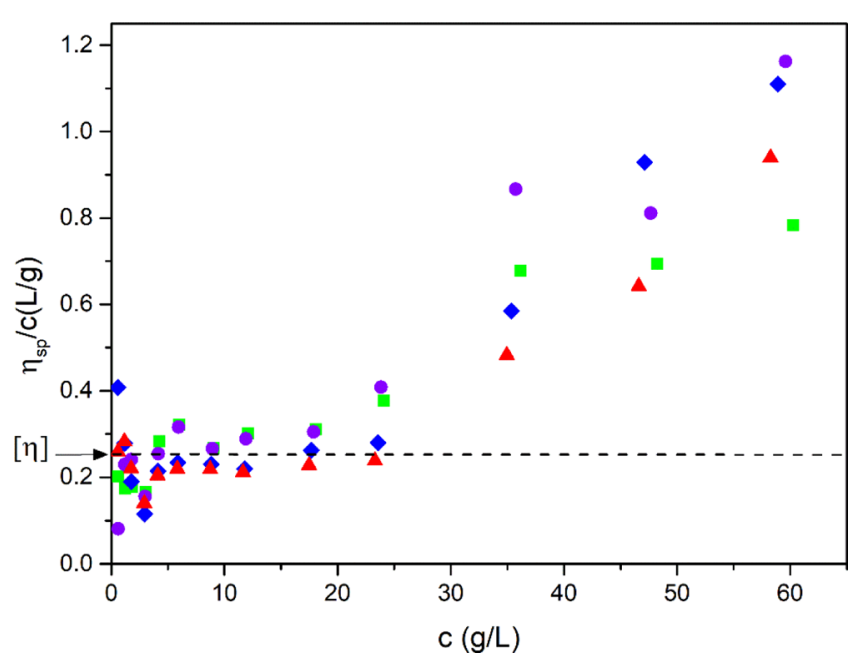

Figure 9. Specific viscosity $\eta_{\mathrm{sp}}[\mathrm{L} / \mathrm{g}]$ scaled by the cellulose concentration $c[\mathrm{~g} / \mathrm{L}]$, vs $c$ deduced from zero-shear viscosities extrapolated from flow curves shown in Figure 8, for a range of temperatures: $20{ }^{\circ} \mathrm{C}$ (green-filled squares), $40{ }^{\circ} \mathrm{C}$ (purple-filled circles), $60{ }^{\circ} \mathrm{C}$ (dark blue-filled diamonds), $80{ }^{\circ} \mathrm{C}$ (red-filled triangles). The intercept of the curves with the $y$-axis is the intrinsic viscosity $[\eta]$, which is estimated to be $[\eta] \approx 0.25 \mathrm{~L} / \mathrm{g}$ (dashed line), roughly independent of temperature.

$[\eta]$, which for fully dissolved polymer chains is a measure for the chain swelling. The curves show significant scatter; but within the margin of error, the intercept with the $y$-axis does not appear to depend on temperature, such that we arrive at an estimated, temperature-independent intrinsic viscosity $[\eta] \approx$ $0.25 \mathrm{~L} / \mathrm{g}$. The primary component in new ternary solvent system is still NMMO monohydrate. To simplify, we assume that the Kuhn-Mark-Houwink relationship in our case is equivalent to that of in pure NMMO monohydrate which reported about fibrous cellulose dissolved in pure NMMO monohydrate by Eckelt et al. ${ }^{44}$ The molar mass of MCC was estimated by following the equation

$$
\log \left([\eta] / \mathrm{mL} \mathrm{g}^{-1}\right)=-1.465+0.735 \log M
$$

Using the above equation, we get a reasonable estimation of the $M_{\mathrm{w}}$ of the cellulose. The molar mass of MCC is reported in Table 3.

The upturn of the curves at higher concentrations, indicative for the start of the semi-dilute regime of overlapping polymer chains, occurs at concentrations on the order of the inverse intrinsic viscosity, as it should. As shown in Figure 10, at higher concentrations, the solutions become viscoelastic, with frequency-dependent storage and loss moduli characteristic for semi-dilute solutions. ${ }^{43}$

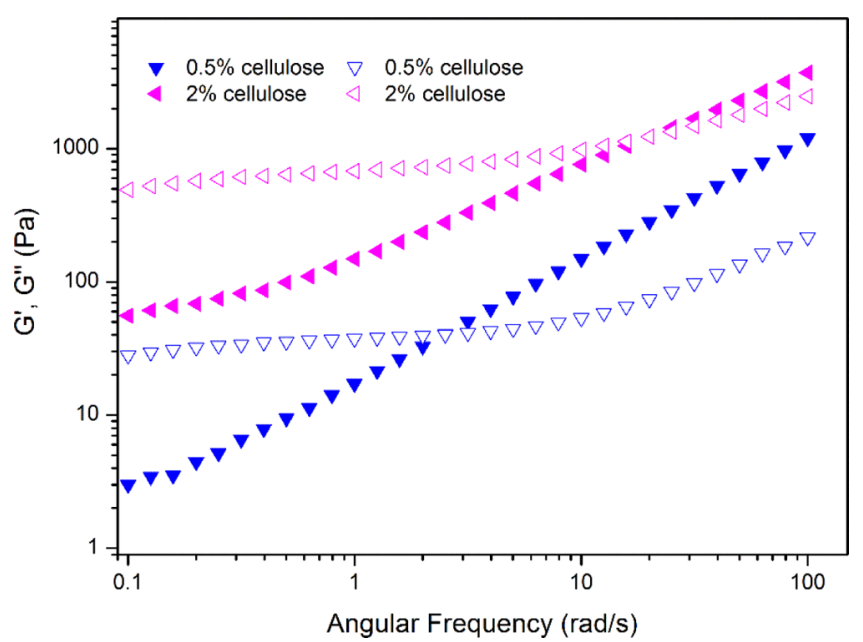

Figure 10. Frequency sweeps for semidilute solutions of microscrystalline cellulose in NCM25\% ternary solvent at $20^{\circ} \mathrm{C}$. Storage modulus $G^{\prime}(\omega)$ (open points) and loss modulus $G^{\prime \prime}(\omega)$ (filled points) as a function of the angular frequency $\omega$. Cellulose concentrations: $0.5 \%$ wt $\approx 6.03 \mathrm{~g} / \mathrm{L}$ (blue, downward-pointing triangles), $2 \%$ wt $\approx 24.12 \mathrm{~g} / \mathrm{L}$ (pink, left-pointing triangles).

In summary, the rheological characterization demonstrates that the MCC has fully dissolved in the NCM25\% solvent and that it is a good solvent for cellulose over the temperature range of $20-80{ }^{\circ} \mathrm{C}$ investigated here.

One specific regeneration application that we are interested in is the formation of various soft cellulose microstructures of controlled morphology by using antisolvent precipitation. The morphology of microstructures obtained by antisolvent precipitation depends on both thermodynamic and kinetics parameters, such as the depth of the quench in terms of solvent quality, the viscosity, mixing conditions, and so forth. ${ }^{45}$ and can vary from spheroidal ${ }^{46}$ to rod-like ${ }^{47}$ to nanofiber, ${ }^{48}$ ribbonlike, sheet or dendritic structures. ${ }^{45}$ If successfully prepared, such soft polymeric microstructures can be utilized in encapsulation and delivery of active components for pharmaceutical and food applications, that is,. as bulk rheology modifiers and structuring agents as well as surface property modifiers, nonwoven scaffolds for air/water filtration, affinity separation and biomedical applications.

Here we merely demonstrate that very different cellulose microstructural particles are obtained by precipitating in antisolvents with very different viscosities, particularly water and glycerol/water (70/30 wt \%). The cellulose solution was injected into the antisolvent under the stirring condition of about $800 \mathrm{rpm}$. Sample was then continued stirring for further $15 \mathrm{~min}$. Centrifugation was carried out several times to remove the solvent and antisolvents. CLSM images of the resulting microstructures are shown in Figure $11 \mathrm{~b}, \mathrm{c}$. For the low viscosity antisolvent (water) we obtain flake-, or sheet-like

Table 3. Characteristics of Original Microcrystalline Cellulose and Cellulose Regenerated via Antisolvent Precipitation in Water and Glycerol/Water (70/30 wt \%) Mixture

\begin{tabular}{|c|c|c|c|c|c|c|}
\hline \multirow[b]{2}{*}{ materials } & \multirow[b]{2}{*}{ molar mass $(\mathrm{kg} / \mathrm{mol})$} & \multirow[b]{2}{*}{ CrI (\%) } & \multicolumn{2}{|c|}{$d$-spacing $(\mathrm{nm})$} & \multicolumn{2}{|c|}{ crystallite size $(\mathrm{nm})$} \\
\hline & & & $d_{200}$ & $d_{020}$ & $L_{200}$ & $L_{020}$ \\
\hline original MCC & 180 & 75.2 & 0.3934 & & 6.8945 & \\
\hline cellulose regenerated by water & & 54.6 & & 0.4312 & & 2.2332 \\
\hline cellulose regenerated by glycerol/water $(70 / 30 \mathrm{wt} \%)$ mixture & & 50.0 & & 0.4307 & & 1.6025 \\
\hline
\end{tabular}


(a)

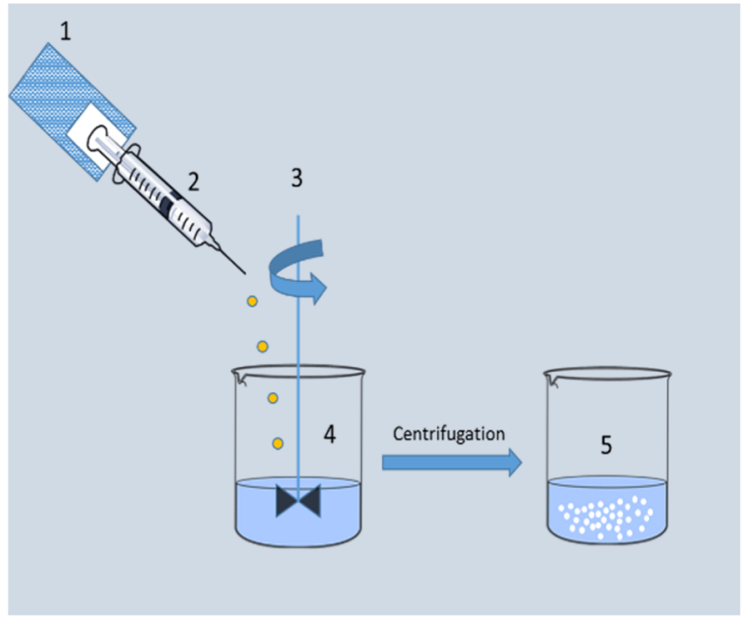

(b)

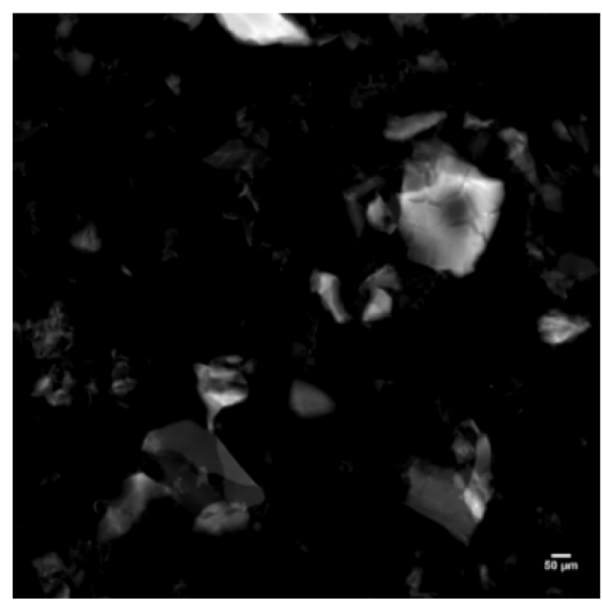

(c)

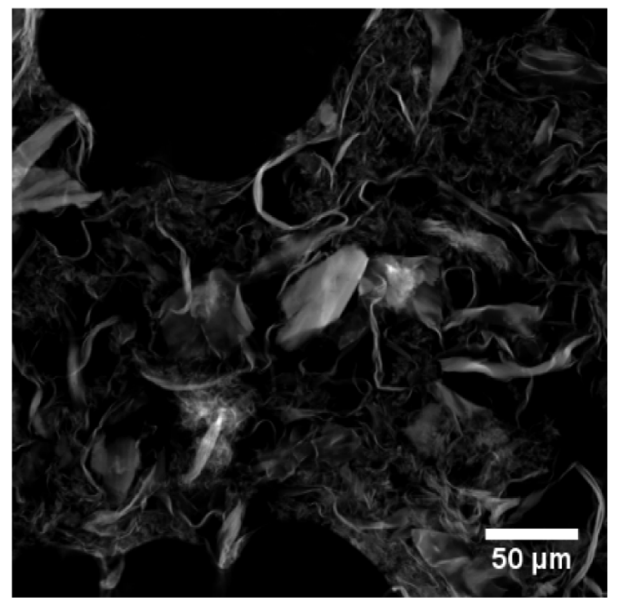

Figure 11. (a) Schematic diagram of antisolvent precipitation set-up (1) pump-(2) cellulose solution in NCM25\% solvent-(3) propeller stirrer-(4) antisolvent-(5) regenerated cellulose suspension; CLSM images of the regenerated cellulose (labeled with Calcofluor white stain) precipitated by different type of antisolvents, (b) prepared b by water as antisolvent; (c) prepared by glycerol-water mixture as antisolvent. All samples were in wet state.

microstructure, whereas for the high viscosity antisolvent, we obtain fiber-like structures of regenerated cellulose. It could be due to the difference in the diffusion coefficient of used antisolvent, which in turn changes the precipitation rate of cellulose, leading to skin formation in the case of fast mixing and more elongated structures in the case of slow mixing. We expect that the rapid antisolvent precipitation leads to a local packing of the cellulose chains that is mostly amorphous. Indeed, this phenomenon is pointed out by the XRD results shown in Figure S4. From the XRD spectra and eq 1, we estimate the crystallinity index of the original MCC, and the regenerated cellulose obtained the low- and high viscosity antisolvents. The lattice spacing (d-spacing) $\left(d_{h, k, l}\right)$ and the crystallite sizes $\left(L_{h, k, l}\right)$ of native cellulose and regenerated cellulose are calculated using eqs 2 and 3, respectively. All of these results are summarized in Table 3.

The XRD pattern of native MCC exhibits the typical diffraction peaks of cellulose $\mathrm{I} \beta$, that is, peaks at around $2 \theta \approx$ $15,16.4$, and $22.6^{\circ}$, which corresponds to diffraction planes of $\left(\begin{array}{lll}1 & -1 & 0\end{array}\right),\left(\begin{array}{lll}1 & 1 & 0\end{array}\right)$, and $\left(\begin{array}{lll}2 & 0 & 0\end{array}\right)$, respectively. ${ }^{49}$ The XRD diffraction patterns of the cellulose regenerated via antisolvent precipitation shows broad peaks at $2 \theta \approx 20.2,21.8^{\circ}$ plus a peak of lower intensity at $2 \theta \approx 12.1^{\circ}$, corresponding to the (lll 110$)$,

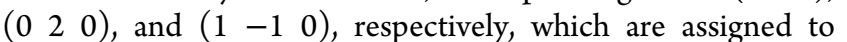
cellulose II. ${ }^{50}$ These characteristics are in good agreement with previous studies in which cellulose was dissolved in ionic liquids. ${ }^{51,52}$ To be specific, there is a slight difference between the cases of antisolvent precipitation in a low and a high viscosity antisolvent. The difference is from the diffraction of $\left(\begin{array}{lll}1 & -1 & 0\end{array}\right)$ lattice plane intensity. The $\left(\begin{array}{lll}1 & -1 & 0\end{array}\right)$ diffraction intensity is stronger when coagulated in water and less strong when coagulated in glycerol/water mixture. It is probably due to the preferential growth of this plane in different coagulant. ${ }^{53}$

From the calculated data summarized in Table 3, CrI tend to decrease from $75.2 \%$ in native cellulose to 54.6 and $50.0 \%$ in regenerated cellulose in water and glycerol/water mixture, respectively. The reduction of crystallinity suggests that the cellulose structure become more disordered after the regeneration process. We expect that the rapid antisolvent precipitation leads to a local packing of the cellulose chains 
that is mostly amorphous. Indeed, this phenomenon is pointed out by the XRD results shown in Figure S4. In addition, the crystallite sizes showed a decrease trend in those of regenerated cellulose compared to native cellulose which also confirm the formation of the amorphous cellulose structure after the regeneration process.

Recycling of Cellulose Ternary Solvent. NMMO technology is well-known for its high solvent recovery efficiency. In this study, we investigated the recovery yield of ternary solvent NCM25\% after antisolvent precipitation process. The ternary solvent was recycled five times with the recovery yields are shown in Figure S5. In general, the efficiency of the solvent recovery process exceeds $95 \%$ after 5 times. It is noticeable to mention that the solvent, after being recycled, retains the same solubility of cellulose (of 14\% wt cellulose) as the original solvent.

The water content was monitored after each cycle. There is a decreasing trend of water content in the solvent mixture. This can be explained by the loss of water that occurs by evaporation during recovery process. In order to upgrade the production process on an industrial scale, the control and monitor of the water content in the solvent mixture need to be optimized.

The chemical composition of cellulose solvent NCM25\% and that of after 5 recycling times were further characterized using FT-IR, as shown in Figure S6. As seen, there were no obvious differences between these two spectra, supporting the chemical stability of our new cellulose solvent system after the whole process of dissolution and regeneration. The fact that we have used a relatively pure cellulose source such as MCC and our studied cellulose solvent system has a very low vapor pressure at recycling conditions, are also important. However, when uses less pure cellulose sources or different recycling processes, this might change because of the accumulation of impurities in the solvent during the recycling and/or some additional changes due to chemical reactions.

\section{CONCLUSIONS}

In summary, we have outlined a novel, green chemistry-based approach for the preparation of new cellulose solvents, based on mixtures of a NADES and a primary cellulose solvent. Because of the strong hydrogen-bond donor and acceptor ability of both the NADES and the primary cellulose solvent, the resulting system could be a cellulose solvent. The new ternary system showed an improved cellulose dissolution capacity when compared to the "pure" solvents and reduced the melting point. To perform effectively, both NADES and the cellulose solvent need to be chemically compatible, with no-chemical reaction occurring between them and they should be able to form a synergistic eutectic mixture. To reveal the exact design rules for this approach to work will require some further studies, but we believe that this is a generic approach, which allows engineering a whole new class of green and mild cellulose solvent systems and the creation of a vast number of new cellulose-based (soft) materials.

To illustrate the feasibility of this approach, we have demonstrated that the NADES CC/MA (CM) is a very effective cosolvent for NMMO. Specifically, for the ternary solvent NMMO monohydrate + 25\% CM1.5:1, NCM25\%, the crystallization of NMMO monohydrate is completely prevented so that it can be used over a much broader range of temperatures. The ternary solvent system NCM25\% showed an improvement on the cellulose solubility. The solvent system can be recycled with recovery yields up to $95 \%$ and still retain the ability to dissolve cellulose. This is a clear demonstration of high applicability of this ternary solvent system in industry.

From rheological data, we conclude that in the new ternary solvent, cellulose is truly molecularly dissolved, and the solvent quality remains constant over the range of temperatures between 20 and $80{ }^{\circ} \mathrm{C}$ (where pure NMMO monohydrate is solid). We have also shown that the new solvent system allows the facile production of highly anisotropic, soft cellulose particles via antisolvent precipitation.

\section{ASSOCIATED CONTENT}

\section{Supporting Information}

The Supporting Information is available free of charge at https://pubs.acs.org/doi/10.1021/acssuschemeng.0c04982.

${ }^{1} \mathrm{H}$ NMR spectra of CC-MA NADES (CM1.5-1) after preparation and after 2 weeks stored at ambient conditions, ${ }^{13} \mathrm{C}$ NMR spectra of original MA, CC, and CC/MA NADES (CM1.5-1) after 2 weeks stored at ambient conditions, DSC thermograms of ternary cellulose solvent NCM25\% and 2\% wt MCC dissolved in NCM25\%, X-ray diffraction pattern of native cellulose and regenerated cellulose, recovery yield in $\%$ and water content (\%) of ternary cellulose solvent NCM25\%, and FT-IR spectra of ternary cellulose solvent NCM25\% before and NCM25\% after 5 recycle times (PDF)

\section{AUTHOR INFORMATION}

\section{Corresponding Author}

Simeon D. Stoyanov - Laboratory of Physical Chemistry and Soft Matter, Agrotechnology \& Food Sciences Group, Wageningen UR, Wageningen 6708WE, The Netherlands; Department of Chemical and Biomolecular Engineering, North Carolina State University, Raleigh, North Carolina 27695, United States; Department of Mechanical Engineering, University College London, London WC1E 7JE, U.K.; Email: Simeon.Stoyanov@wur.nl

\section{Authors}

Huy Vu Duc Nguyen - Laboratory of Physical Chemistry and Soft Matter, Agrotechnology \& Food Sciences Group, Wageningen UR, Wageningen 6708WE, The Netherlands; (1) orcid.org/0000-0002-5086-649X

Renko De Vries - Laboratory of Physical Chemistry and Soft Matter, Agrotechnology \& Food Sciences Group, Wageningen UR, Wageningen 6708WE, The Netherlands; 10 orcid.org/ 0000-0001-8664-3135

Complete contact information is available at: https://pubs.acs.org/10.1021/acssuschemeng.0c04982

\section{Notes}

The authors declare no competing financial interest.

\section{ACKNOWLEDGMENTS}

Part of this work has been financially supported by Unilever Research in The Netherlands and by the Graduate School VLAG, The Netherlands.

\section{REFERENCES}

(1) Klemm, D.; Heublein, B.; Fink, H.-P.; Bohn, A. Cellulose: fascinating biopolymer and sustainable raw material. Angew. Chem., Int. Ed. 2005, 44, 3358-3393. 
(2) Fink, H.-P.; Weigel, P.; Purz, H. J.; Ganster, J. Structure formation of regenerated cellulose materials from NMMO-solutions. Prog. Polym. Sci. 2001, 26, 1473-1524.

(3) Matsumoto, T.; Tatsumi, D.; Tamai, N.; Takaki, T. Solution properties of celluloses from different biological origins in $\mathrm{LiCl}$ DMAc. Cellulose 2001, 8, 275-282.

(4) Fischer, S.; Leipner, H.; Thümmler, K.; Brendler, E.; Peters, J. Inorganic molten salts as solvents for cellulose. Cellulose 2003, 10, 227-236.

(5) Innerlohinger, J.; Weber, H. K.; Kraft, G. Aerocellulose: Aerogels and Aerogel-like Materials Made from Cellulose. Macromol. Symp. 2006, 244, 126-135.

(6) Krysztof, M.; Olejnik, K.; Kulpinski, P.; Stanislawska, A.; Khadzhynova, S. Regenerated cellulose from N-methylmorpholine Noxide solutions as a coating agent for paper materials. Cellulose 2018, $25,3595-3607$.

(7) Rosenau, T.; Potthast, A.; Sixta, H.; Kosma, P. The chemistry of side reactions and byproduct formation in the system NMMO/ cellulose (Lyocell process). Prog. Polym. Sci. 2001, 26, 1763-1837.

(8) Henry, C. L. Use of Co-solvents in Amine N-Oxide Solutions. U.S. Patent 4,290,815 A, Sep 22, 1981. https://patents.google.com/ patent/US4290815A/en.

(9) Freyburger, A. Novel Bio-Based Materials from Cellulose and Chitin. Ph.D Thesis, University of Regensburg, April 2018. https:// epub.uni-regensburg.de/37181/.

(10) Freyburger, A.; Werner, K.; Zollfrank, C.; Duan, Y. Process for the Preparation of a Cellulose Product. WO 2017211798 A1, Dec 14, 2017. https://patentscope.wipo.int/search/en/detail.jsf?docId= WO2017211798.

(11) Zaikov, G., Chemistry of Polysaccharides; CRC Press, 2005.

(12) Gunnars, S.; Wågberg, L.; Cohen Stuart, M. A. Model films of cellulose: I. Method development and initial results. Cellulose 2002, 9, 239-249.

(13) Abbott, A. P.; Boothby, D.; Capper, G.; Davies, D. L.; Rasheed, R. K. Deep eutectic solvents formed between choline chloride and carboxylic acids: versatile alternatives to ionic liquids. J. Am. Chem. Soc. 2004, 126, 9142-9147.

(14) Paiva, A.; Craveiro, R.; Aroso, I.; Martins, M.; Reis, R. L.; Duarte, A. R. C. Natural deep eutectic solvents-solvents for the 21st century. ACS Sustainable Chem. Eng. 2014, 2, 1063-1071.

(15) Liu, Y.; Friesen, J. B.; McAlpine, J. B.; Lankin, D. C.; Chen, S.N.; Pauli, G. F. Natural deep eutectic solvents: properties, applications, and perspectives. J. Nat. Prod. 2018, 81, 679-690.

(16) Francisco, M.; van den Bruinhorst, A.; Kroon, M. C. New natural and renewable low transition temperature mixtures (LTTMs): screening as solvents for lignocellulosic biomass processing. Green Chem. 2012, 14, 2153-2157.

(17) Procentese, A.; Johnson, E.; Orr, V.; Garruto Campanile, A.; Wood, J. A.; Marzocchella, A.; Rehmann, L. Deep eutectic solvent pretreatment and subsequent saccharification of corncob. Bioresour. Technol. 2015, 192, 31-36.

(18) Sharma, M.; Mukesh, C.; Mondal, D.; Prasad, K. Dissolution of $\alpha$-chitin in deep eutectic solvents. RSC Adv. 2013, 3, 18149-18155.

(19) Medronho, B.; Lindman, B. Brief overview on cellulose dissolution/regeneration interactions and mechanisms. Adv. Colloid Interface Sci. 2015, 222, 502-508.

(20) Lindman, B.; Karlström, G.; Stigsson, L. On the mechanism of dissolution of cellulose. J. Mol. Liq. 2010, 156, 76-81.

(21) Liu, Y.; Guo, B.; Xia, Q.; Meng, J.; Chen, W.; Liu, S.; Wang, Q.; Liu, Y.; Li, J.; Yu, H. Efficient cleavage of strong hydrogen bonds in cotton by deep eutectic solvents and facile fabrication of cellulose nanocrystals in high yields. ACS Sustainable Chem. Eng. 2017, 5, $7623-7631$.

(22) Lynam, J. G.; Kumar, N.; Wong, M. J. Deep eutectic solvents' ability to solubilize lignin, cellulose, and hemicellulose; thermal stability; and density. Bioresour. Technol. 2017, 238, 684-689.

(23) Satlewal, A.; Agrawal, R.; Bhagia, S.; Sangoro, J.; Ragauskas, A. J. Natural deep eutectic solvents for lignocellulosic biomass pretreatment: recent developments, challenges and novel opportunities. Biotechnol. Adv. 2018, 36, 2032.

(24) Castro, V. I. B.; Mano, F.; Reis, R. L.; Paiva, A.; Duarte, A. R. C. Synthesis and Physical and Thermodynamic Properties of Lactic Acid and Malic Acid-Based Natural Deep Eutectic Solvents. J. Chem. Eng. Data 2018, 63, 2548.

(25) Zhang, Q.; De Oliveira Vigier, K.; Royer, S.; Jérôme, F. Deep eutectic solvents: syntheses, properties and applications. Chem. Soc. Rev. 2012, 41, 7108-7146.

(26) Lee, J. A.; Ahn, J. H.; Lee, S. Y. Organic Acids: Succinic and Malic Acids. Comprehensive Biotechnology; Pergamon, 2019; pp 172187.

(27) Segal, L.; Creely, J. J.; Martin, A. E., Jr.; Conrad, C. M. An empirical method for estimating the degree of crystallinity of native cellulose using the X-ray diffractometer. Text. Res. J. 1959, 29, 786794.

(28) Nam, S.; French, A. D.; Condon, B. D.; Concha, M. Segal crystallinity index revisited by the simulation of $\mathrm{X}$-ray diffraction patterns of cotton cellulose $\mathrm{I} \beta$ and cellulose II. Carbohydr. Polym. 2016, 135, 1-9.

(29) Ju, X.; Bowden, M.; Brown, E. E.; Zhang, X. An improved X-ray diffraction method for cellulose crystallinity measurement. Carbohydr. Polym. 2015, 123, 476-481.

(30) Rodriguez Rodriguez, N.; van den Bruinhorst, A.; Kollau, L. J. B. M.; Kroon, M. C.; Binnemans, K. Degradation of deep-eutectic solvents based on choline chloride and carboxylic acids. ACS Sustainable Chem. Eng. 2019, 7, 11521-11528.

(31) Gorman, M. The evidence from infrared spectroscopy for hydrogen bonding: A case history of the correlation and interpretation of data. J. Chem. Educ. 1957, 34, 304.

(32) Perkins, S. L.; Painter, P.; Colina, C. M. Experimental and computational studies of choline chloride-based deep eutectic solvents. J. Chem. Eng. Data 2014, 59, 3652-3662.

(33) Dai, Y.; Witkamp, G.-J.; Verpoorte, R.; Choi, Y. H. Tailoring properties of natural deep eutectic solvents with water to facilitate their applications. Food Chem. 2015, 187, 14-19.

(34) Abbott, A. P.; Capper, G.; Davies, D. L.; Rasheed, R. K.; Tambyrajah, V. Novel solvent properties of choline chloride/urea mixtures. Chem. Commun. 2003, 7, 70-71.

(35) Fischer, V.; Kunz, W. Properties of sugar-based low-melting mixtures. Mol. Phys. 2014, 112, 1241-1245.

(36) Gale, E.; Wirawan, R. H.; Silveira, R. L.; Pereira, C. S.; Johns, M. A.; Skaf, M. S.; Scott, J. L. Directed discovery of greener cosolvents: New cosolvents for use in ionic liquid based organic electrolyte solutions for cellulose dissolution. ACS Sustainable Chem. Eng. 2016, 4, 6200-6207.

(37) Biganska, O.; Navard, P.; Bédué, O. Crystallisation of cellulose/ $\mathrm{N}$-methylmorpholine-N-oxide hydrate solutions. Polymer 2002, 43, 6139-6145.

(38) Liu, R.-G.; Shen, Y.-Y.; Shao, H.-L.; Wu, C.-X.; Hu, X.-C. An analysis of Lyocell fiber formation as a melt-spinning process. Cellulose 2001, 8, 13-21.

(39) Cuissinat, C.; Navard, P. Swelling and Dissolution of Cellulose Part 1: Free Floating Cotton and Wood Fibres in N-Methylmorpholine-N-oxide-Water Mixtures. Macromol. Symp. 2006, 244, 1-18.

(40) Eckelt, J.; Eich, T.; Röder, T.; Rüf, H.; Sixta, H.; Wolf, B. A. Phase diagram of the ternary system NMMO/water/cellulose. Cellulose 2009, 16, 373-379.

(41) Sescousse, R.; Le, K. A.; Ries, M. E.; Budtova, T. Viscosity of cellulose- imidazolium-based ionic liquid solutions. J. Phys. Chem. B 2010, 114, 7222-7228.

(42) Fendt, S.; Padmanabhan, S.; Blanch, H. W.; Prausnitz, J. M. Viscosities of acetate or chloride-based ionic liquids and some of their mixtures with water or other common solvents. J. Chem. Eng. Data 2011, 56, 31-34.

(43) Doi, M.; Edwards, S. F. The Theory of Polymer Dynamics; Oxford University Press, 1988; Vol. 73.

(44) Eckelt, J.; Knopf, A.; Röder, T.; Weber, H. K.; Sixta, H.; Wolf, B. A. Viscosity-molecular weight relationship for cellulose solutions in 
either NMMO monohydrate or cuen. J. Appl. Polym. Sci. 2011, 119, 670-676.

(45) Roh, S.; Williams, A. H.; Bang, R. S.; Stoyanov, S. D.; Velev, O. D. Soft dendritic microparticles with unusual adhesion and structuring properties. Nat. Mater. 2019, 18, 1315.

(46) Richter, A. P.; Brown, J. S.; Bharti, B.; Wang, A.; Gangwal, S.; Houck, K.; Cohen Hubal, E. A.; Paunov, V. N.; Stoyanov, S. D.; Velev, O. D. An environmentally benign antimicrobial nanoparticle based on a silver-infused lignin core. Nat. Nanotechnol. 2015, 10, 817.

(47) Alargova, R. G.; Bhatt, K. H.; Paunov, V. N.; Velev, O. D. Scalable synthesis of a new class of polymer Microrods by a liquidliquid dispersion technique. Adv. Mater. 2004, 16, 1653-1657.

(48) Smoukov, S. K.; Tian, T.; Vitchuli, N.; Gangwal, S.; Geisen, P.; Wright, M.; Shim, E.; Marquez, M.; Fowler, J.; Velev, O. D. Scalable liquid shear-driven fabrication of polymer nanofibers. Adv. Mater. 2015, 27, 2642-2647.

(49) Nishiyama, Y.; Langan, P.; Chanzy, H. Crystal structure and hydrogen-bonding system in cellulose $\mathrm{I} \beta$ from synchrotron $\mathrm{X}$-ray and neutron fiber diffraction. J. Am. Chem. Soc. 2002, 124, 9074-9082.

(50) Kolpak, F. J.; Blackwell, J. Determination of the structure of cellulose II. Macromolecules 1976, 9, 273-278.

(51) Sirviö, J. A. Fabrication of regenerated cellulose nanoparticles by mechanical disintegration of cellulose after dissolution and regeneration from a deep eutectic solvent. J. Mater. Chem. A 2019, 7, 755-763.

(52) Tang, S.; Baker, G. A.; Ravula, S.; Jones, J. E.; Zhao, H. PEGfunctionalized ionic liquids for cellulose dissolution and saccharification. Green Chem. 2012, 14, 2922-2932.

(53) Isobe, N.; Kim, U.-J.; Kimura, S.; Wada, M.; Kuga, S. Internal surface polarity of regenerated cellulose gel depends on the species used as coagulant. J. Colloid Interface Sci. 2011, 359, 194-201. 